ORIGINAL ARTICLE

\title{
Are routine iron stains on bone marrow trephine biopsy specimens necessary?
}

\author{
S E Stuart-Smith, D A Hughes, B J Bain
}

J Clin Pathol 2005;58:269-272. doi: 10.1136/jcp.2004.017038

See end of article for authors' affiliations .....................

Correspondence to: Dr S E Stuart-Smith, Department of

Haematology, St Thomas' Hospital, Lambeth Palace Road, London SE1 7EH, UK; sarass@tinyworld.co. uk

Accepted for publication 8 July 2004

\begin{abstract}
Aims: To determine the role of Perls' staining in bone marrow trephine biopsy sections.
Methods: The haemosiderin content of 155 Perls' stained, formic acid decalcified trephine biopsy sections was assessed and compared with Perls' stained aspirate samples in 105 cases and haematoxylin and eosin (H\&E) stained biopsy sections in all cases.

Results: An evaluable aspirate film with positive iron or at least seven negative particles was available for 105 biopsies. Only 71 of 95 cases with detectable aspirate iron had haemosiderin detectable on a Perls' stained section. None of 10 samples with a negative aspirate had a positive trephine biopsy. Haemosiderin was positive in 101 of the 155 Perls' stained sections, and was detectable on the H\&E stained section in 71 of these cases. In five of 54 cases with negative Perls' staining, a small amount of haemosiderin was thought to be present on H\&E staining.

Conclusions: Aspirate smears reflect bone marrow iron stores more reliably than formic acid decalcified trephine biopsy sections. The presence of iron in Perls' stained aspirates in $44 \%$ of cases with negative Perls' stained sections indicates that iron is often lost from sections during decalcification. However, $61 \%$ of cases with unassessable aspirate samples had a positive trephine biopsy Perls' stain, contributing useful clinical information about iron status. Preparation of Perls' stained sections only in cases in which aspirate samples are inadequate for iron assessment and no obvious haemosiderin is present in an H\&E stained section could produce savings in staff time and reagent costs.
\end{abstract}

"The place of a Perls' stain of trephine biopsy sections is less well defined"

\section{METHODS}

One hundred and fifty five consecutive bone marrow biopsy specimens taken at St Mary's Hospital, London, UK, over a period of one year, from August 2001, were examined. Samples were from a wide range of patients with clinical diagnoses including unexplained anaemia, multiple myeloma, myelodysplastic syndrome, acute and chronic leukaemia, and lymphoma.

Sections were fixed and then decalcified overnight in a decalcifying solution ( $1000 \mathrm{ml}$ formic acid, $3250 \mathrm{ml}$ distilled water, and $250 \mathrm{ml}$ formaldehyde). Samples were rinsed in distilled water and incubated for 20 minutes in a solution of equal parts of $2 \%$ hydrochloric acid and $2 \%$ potassium ferrocyanide, before rinsing in distilled water then tap water, and finally counterstaining in $1 \%$ aqueous neutral red. The haemosiderin and iron content of H\&E and Perls' stained bone marrow trephine biopsy specimens was graded using the system applied by Jakkunen to bone marrow aspirate samples $^{2}$ (table 1).

Table 1 Grading of iron or haemosiderin in bone marrow biopsy sections ${ }^{2}$

stain may be unreliable and potentially misleading because or all of the iron may have been removed during decalcification. Because golden brown refractile haemosiderin is often apparent on a haematoxylin and eosin (H\&E) stained trephine biopsy section, we also investigated whether an H\&E stain could give the same information as a Perls' stain. On the basis of our findings, we devised an algorithm for an economical assessment of the presence or absence of haemosiderin in a trephine biopsy specimen.
Abbreviations: $H \& E$, haematoxylin and eosin 


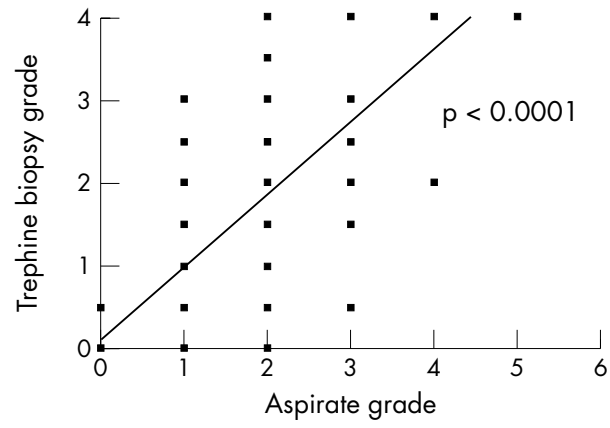

Figure 1 Comparison of iron grading by Perls' staining of bone marrow aspirates and trephine biopsies.

Each H\&E or Perls' stained section was examined for two minutes by two independent observers and a grade was assigned for the amount of haemosiderin. To avoid bias, the H\&E stained section was examined before the Perls' stain. Care was taken to distinguish between formalin pigment and haemosiderin, formalin pigment being deep brown to black and randomly distributed, whereas haemosiderin is golden brown when unstained and deep blue in a Perls' stain, and is related to tissue structures. The observers were blinded to the results of serological iron studies, the patient's medical condition, the results of Perls' staining of aspirate films, and the assessment of the second observer. For each patient with an assessable aspirate, a comparison was made between the grading of iron by a Perls' stain of the aspirate, a Perls' stain of the marrow section, and an H\&E stain of the biopsy section.

A bone marrow aspirate was considered assessable if at least seven bone marrow fragments were available for assessment and all were negative, or if there was at least one positive fragment present. Aspirate films that had fewer than seven fragments, none of which showed positive staining for iron, were considered unassessable. ${ }^{1}$ The evaluation of iron stores was carried out in bone marrow sections from all patients without assessable aspirates, to determine the value of assessment of haemosiderin on an H\&E stain or a Perls' stain in cases that could not otherwise be evaluated.

\section{RESULTS}

In $105(68 \%)$ of the patients, an assessable aspirate sample was available for comparison with the trephine biopsy specimen. The Perls' stain of the trephine biopsy section was always negative when the aspirate was negative, but it failed to detect haemosiderin in a quarter of patients with a positive aspirate (table 2). Despite the lower sensitivity, there was a significant correlation between the assessment of iron stores in trephine biopsy sections and aspirates $(\mathrm{p}<0.0001$; $r=0.7$; fig 1).

There were 45 patients $(29 \%)$ in whom the aspirate was unassessable. In 38 patients (25\%), the aspirate sample was

Table 2 Comparison of Perls' staining of trephine biopsy sections and aspirates for 105 patients with an assessable aspirate

\begin{tabular}{lllll}
\hline & $\begin{array}{l}\text { Aspirate } \\
\text { positive }\end{array}$ & & $\begin{array}{l}\text { Aspirate } \\
\text { negative }\end{array}$ \\
\cline { 2 - 3 } & 95 & & 10 \\
\hline Trephine biopsy positive & 71 & 71 & 0 \\
Trephine biopsy negative & 34 & 24 & 10 \\
\hline
\end{tabular}

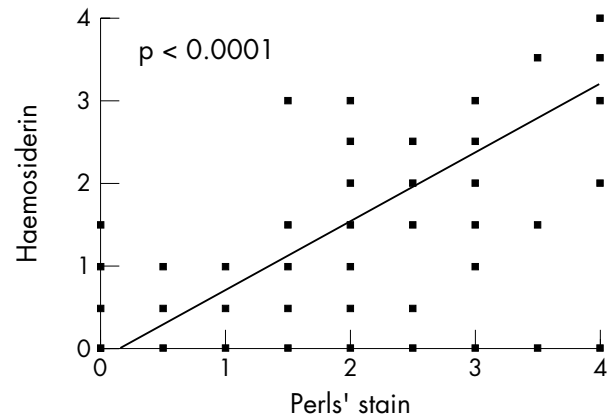

Figure 2 Comparison of grading of iron stores assessed by haemosiderin in haemotoxylin and eosin and Perls' stained bone marrow sections.

aparticulate and haemodilute, there was one case of a blood tap, and in six cases there were six or fewer negative particles. In addition, the haematologist performing the aspirate had not requested an iron stain in three patients, so that no unstained slide was available for staining, and in another two patients an iron stain had been carried out but was not available at the time of our study. Twenty eight of 45 patients with unassessable aspirates had iron stores present on a Perls' stain of a trephine biopsy section, as did two of the five patients for whom no Perls' stained aspirate film was available.

Comparison of Perls' and H\&E staining of trephine biopsy specimens for all 155 cases showed that H\&E staining was less sensitive for the detection of haemosiderin in histological sections and was probably less reliable (table 3 ). There were 30 patients in whom Perls' staining of the trephine biopsy was positive but no haemosiderin was seen on the H\&E stained section. The five cases in which Perls' staining was negative but haemosiderin was thought to be present in an H\&E stained section could represent sampling error, but it is more probable that the detection of a trace of haemosiderin on an H\&E stained section is not reliable. Despite the lower sensitivity, there was a significant correlation between haemosiderin grading of H\&E and Perls' stained sections $(\mathrm{p}<0.0001 ; r=0.82$; fig 2$)$.

The assessment of Perls' staining in trephine biopsy sections was more reproducible than the assessment of haemosiderin in H\&E stained sections when the two observers made independent assessments. Figures 3 and 4 depict the reproducibility of the grading systems for haemosiderin and iron on trephine sections.

On the basis of our results, we devised an algorithm for the selective performance of a Perls' stain on a trephine biopsy section (fig 5). If this algorithm is applied to the current data set, interpreting grade 2 or greater as indicating definite and easily detectable haemosiderin on an H\&E stained section, only 41 of 155 biopsy specimens (23\%) would require a Perls' stain.

Table 3 Comparison of H\&E staining and Perls' staining on trephine biopsy sections

\begin{tabular}{llll}
\hline & $\begin{array}{l}\text { Perls' stain } \\
\text { positive }\end{array}$ & & $\begin{array}{l}\text { Perls' stain } \\
\text { negative }\end{array}$ \\
\cline { 2 - 3 } & $\mathbf{1 0 1}$ & $\mathbf{5 4}$ \\
\hline H\&E stain positive & 76 & 71 & 5 \\
H\&E stain negative & 79 & 30 & 49 \\
\hline H\&E, haematoxylin and eosin. & & \\
\hline
\end{tabular}




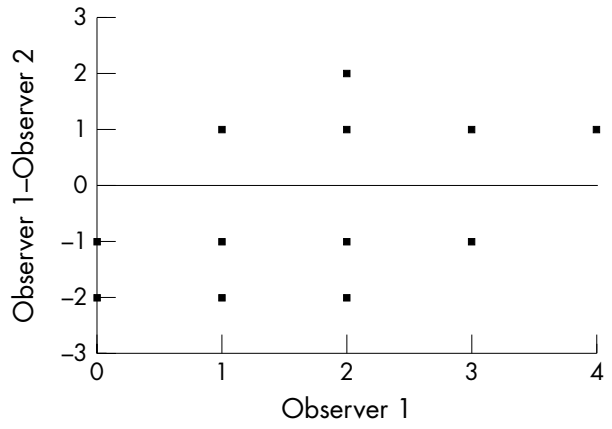

Figure 3 The assessment of bias between two observers grading haemosiderin in haematoxylin and eosin stained bone marrow trephine sections.

\section{DISCUSSION}

In other series in which both aspirates and sections of bone marrow biopsy specimens were examined for the presence of haemosiderin, discrepancies in the assessment of iron stores were evident. Fong et al reported the absence of stainable iron in $8 \%$ of sections in contrast to definite deposits seen in the corresponding aspirate film, and $6 \%$ of sections had significantly less stainable iron than the corresponding films ${ }^{3}$; this American study used decalcification in Zenker's acetic acid solution, a technique not commonly used in the UK or the rest of Europe. Contrary findings were reported by Krause and colleagues, who studied 1000 simultaneously collected aspirate smears and needle biopsies, with the biopsy specimens being placed in Zenker's acetic acid for 15-18 hours and washed for at least eight hours before processing. ${ }^{4}$ They found that when storage iron was present in aspirates, it was always present in the corresponding needle biopsy specimen; the grade of iron stores tended to be lower in films than in sections, leading them to conclude that bone marrow sections were preferable for the evaluation of iron stores. ${ }^{4}$ Neither of these studies specified their criteria for adequacy for assessment of an aspirate film. The series by Krause et al only included specimens in which an aspirate "contained several spicules" (spicule being a term sometimes used to describe a fragment or particle of bone marrow). They did not state the exact number of spicules required. Fong et al stated in their methods that only "technically satisfactory specimens" were used, but did not further define this. We have defined an assessable aspirate precisely as one having either a minimum of seven bone marrow fragments, all of which are negative, or at least one iron positive fragment. ${ }^{1}$

From our study, it is apparent that aspirate films are more sensitive than trephine biopsy sections for the detection of haemosiderin when the biopsy specimens are decalcified in formic acid. They also provide a more accurate reflection of bone marrow iron stores, because decalcification leads to an unquantifiable loss of iron. It is possible that the findings would be different if decalcification were with ethylenediaminetetraacetic acid, which is a less powerful decalcifying agent. For those aspirates that were not evaluable, two thirds of cases had detectable haemosiderin on a Perls' stained biopsy section. There are many reasons for the lack of an assessable aspirate; there may be a total failure to obtain an aspirate in patients with bone marrow fibrosis, or the aspirate may be composed of sinusoidal blood, may clot before films can be made, may be aparticulate, or may contain fewer than seven negative particles. With the increasing use of special tests such as immunophenotyping and cytogenetic and molecular analysis, larger volumes of bone marrow are aspirated. This may contribute to the increased incidence of haemodilute pauciparticulate samples received. Care should

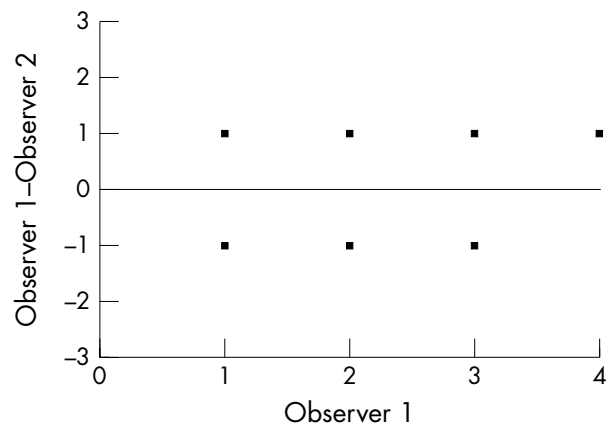

Figure 4 The assessment of bias between two observers grading haemosiderin in Perls' stained bone marrow trephine sections.

be taken to ensure that only a small volume of bone marrow is aspirated into a separate syringe at the start of the procedure for film preparation, before taking a larger volume of marrow for special tests. This would probably lead to a reduction in the number of trephine biopsy specimens requiring an iron stain.

"Aspirate films are more sensitive than trephine biopsy sections for the detection of haemosiderin when the biopsy specimens are decalcified in formic acid"

The scoring of iron status in trephine sections was more reproducible with Perls' staining than with H\&E staining and was also considerably less tedious. Therefore, the examination of H\&E sections to assess haemosiderin cannot replace the Perls' stain, although if plentiful haemosiderin is present

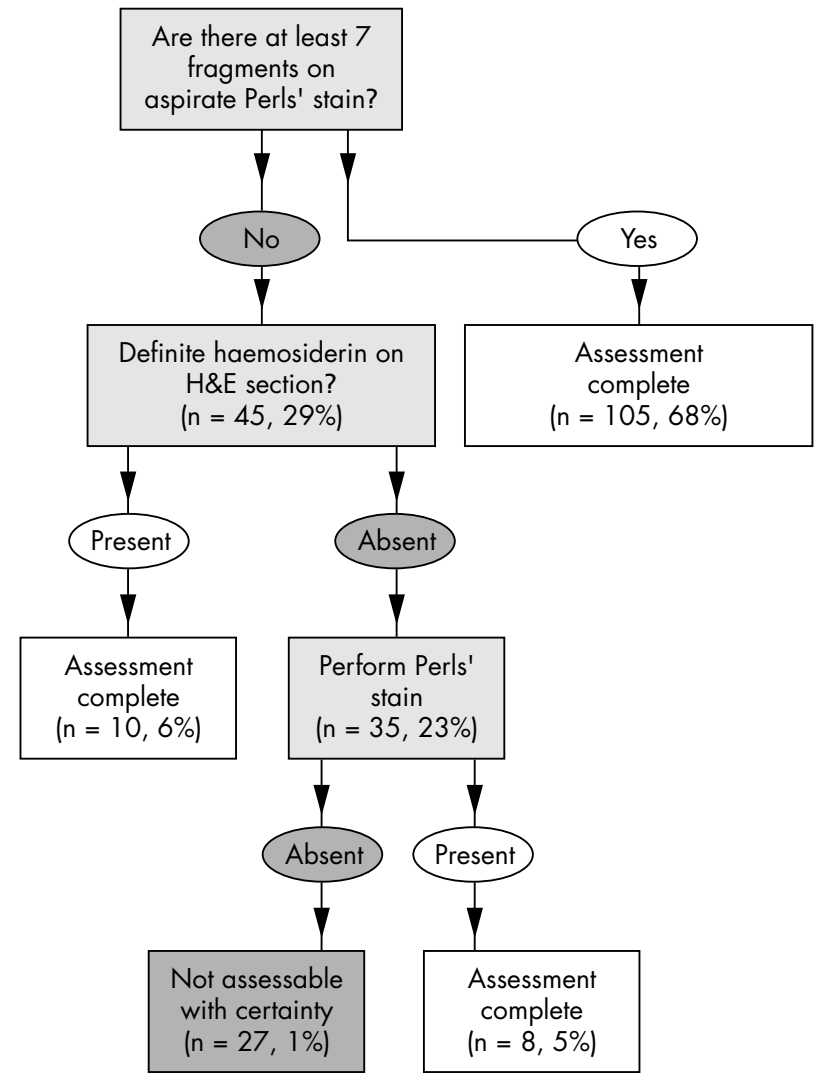

Figure 5 Algorithm for determining whether or not iron is present in the bone marrow, with results of application of this algorithm to our data. $\mathrm{H} \& \mathrm{E}$, haematoxylin and eosin. 


\section{Take home messages}

- Perls' staining of trephine biopsy sections is worthwhile when the bone marrow aspirate is unassessable and there is no obvious haemosiderin in the haematoxylin and eosin stained section

- However, routine Perls' staining of trephine biopsy sections is not necessary

- Not performing a Perls' stain as routine would produce savings in terms of staff time and reagent costs

a Perls' stain is not needed. Because of the loss of iron during decalcification, pathologists need to use appropriate wording when reporting trephine biopsy sections from specimens that have been decalcified. Haemosiderin can be reported as present or increased but not as normal or decreased. A negative Perls' stain should merely be reported as "negative" rather than as showing "absent iron" because this last phrase would be misleading unless further qualified. Similarly, if a Perls' stain is not performed routinely it is useful to report that an H\&E stained section shows haemosiderin to be present or increased. Because of the lack of sensitivity, it is not helpful to comment on the absence of haemosiderin in an H\&E stained section.

We conclude that an iron stain is worthwhile when the aspirate is unassessable and there is no obvious haemosiderin in the H\&E stained section, but that a routine Perls' stain on a trephine biopsy section is not necessary. Not performing a Perls' stain as routine would produce savings in terms of staff time and reagent costs.

\section{ACKNOWLEDGEMENTS}

Histological sections were prepared by the Department of Cellular Pathology at St Mary's Hospital, London, UK.

\section{Authors' affiliations}

S E Stuart-Smith, D A Hughes, B J Bain, Department of Haematology, St Mary's Hospital, Praed Street, London W2 1NY, UK

\section{REFERENCES}

1 Hughes DA, Stuart-Smith SA, Bain BJ. How should stainable iron in bone marrow films be assessed? J Clin Pathol [In press.]

2 Jakkunen $\mathrm{H}$. Iron deficiency in the Finnish adult population. Scand J Haematol 1973;25:1-91

3 Fong TP, Okafor LA, Thomas W, et al. Stainable iron in aspirated and needlebiopsy specimens of marrow: a source of error. Am J Hematol 1977; 1:47-51.

4 Krause JR, Brubaker D, Kaplan S. Comparison of stainable iron in aspirated and needle-biopsy specimens in bone marrow. Am J Clin Pathol 1979;72:68-70.

\section{Register now!}

10th European Forum on Quality Improvement in Health Care

13-15 April 2005, ExCel Conference Centre, London

For further information on how to register please go to:

http://www.quality.bmipg.com 